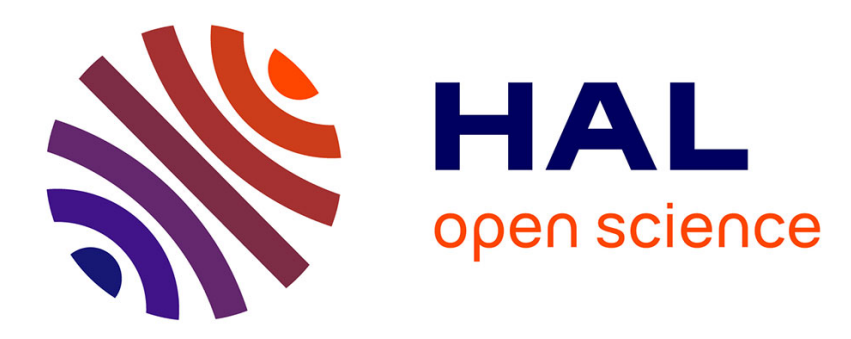

\title{
Synthetic representations of the Poole Frenkel (PF) and Poole regimes
}

\author{
A. Pillonnet, R. Ongaro
}

\section{To cite this version:}

A. Pillonnet, R. Ongaro. Synthetic representations of the Poole Frenkel (PF) and Poole regimes. Revue de Physique Appliquée, 1990, 25 (2), pp.229-242. 10.1051/rphysap:01990002502022900 . jpa00246181

\section{HAL Id: jpa-00246181 https://hal.science/jpa-00246181}

Submitted on 1 Jan 1990

HAL is a multi-disciplinary open access archive for the deposit and dissemination of scientific research documents, whether they are published or not. The documents may come from teaching and research institutions in France or abroad, or from public or private research centers.
L'archive ouverte pluridisciplinaire HAL, est destinée au dépôt et à la diffusion de documents scientifiques de niveau recherche, publiés ou non, émanant des établissements d'enseignement et de recherche français ou étrangers, des laboratoires publics ou privés. 


\title{
Synthetic representations of the Poole Frenkel (PF) and Poole regimes
}

\author{
A. Pillonnet and R. Ongaro \\ Laboratoire d'Electricité, Bât. 721, Université Claude Bernard, Lyon 1, 43 Bd du 11 Novembre 1918, 69622 \\ Villeurbanne Cedex, France
}

(Reçu le 10 novembre 1988, révisé le 17 juillet 1989, accepté le 27 octobre 1989)

\begin{abstract}
Résumé. - Une étude synthétique de l'ionisation de donneurs par un champ électrique est présentée afin d'apporter des éclaircissements, utiles à l'expérimentateur, sur les régimes Poole et Poole Frenkel (PF). L'accent est mis sur la similitude de certaines des hypothèses fondamentales, ainsi que sur le caractère artificiel des hypothèses spécifiques au régime Poole. Quelques ambiguïtés des représentations graphiques rencontrées dans la littérature sont aussi rapportées. De même sont mises en évidence les divergences existant entre les auteurs à propos : de la barrière de potentiel qu'ont à franchir les électrons en bas et en hauts champs ; et de la notion de champ de transition entre les deux régimes. L'exposé est essentiellement consacré au cas des puits coulombiens, bien que des puits à plus court rayon d'action soient aussi évoqués. Deux diagrammes à trois dimensions sont construits, accompagnés de simulations annexes déduites des modèles de Hill, dans le but de délimiter clairement, sur des bases quantitatives, les intervalles de validité respectifs des régimes Poole et PF, ce dernier étant éventuellement assorti d'un effet tunnel assisté thermiquement.
\end{abstract}

\begin{abstract}
A synthetic study of the field-induced ionization of donors is presented in order to bring some insights, usefull to experimentalits, into the Poole and Poole Frenkel (PF) regimes. Emphasis is made on the similarities of some of the fundamental assumptions, as on the artificial character of the hypotheses specific to Poole regime. Some ambiguities dealing with plottings met in literature are also reported. Likewise, discrepancies existing between the authors approaches are put forward, dealing: with the barrier to be overcome by electrons at low and high field strengths; and with the notion of transition field from one regime to the other. The account concerns essentially Coulomb potentials, though the case of more steeper wells is also evoked. Two block diagrams are built up, supplemented by simulations deduced from Hill's models, with the purpose of clear quantitative delimitation of the ranges of validity of Poole and PF regimes, the latter being eventually assorted with a thermally assisted tunnelling.
\end{abstract}

\section{Introduction.}

In a preceding paper [1], we put forward theoretically the existence of a saturation of the Poole Frenkel (PF) effect, at high field strengths. It remains now to review the problems set by an application of the involved model, in the low field range. In this range, at once, initial levelling of current $j_{0}$ versus field $F$ is frequently observed experimentally in a $\left(\lg j_{0} / F\right.$, $F^{1 / 2}$ ) plot. This cannot be accounted for in the simplest PF theories providing for a proportionality of conductivity to $\exp \left(K F^{1 / 2}\right)$. The reduction of such a gap between theory and experiment was attempted by several authors, following three ways. In the first, free charges from a different origin than carriers liberated from donors by the field are assumed to be in excess at low field strengths [2]. Then, no initial field-enhancement of carriers can be expected. Following the second way a reajustment is attempted by introducing a three-dimensional PF effect (PF-3D) (see [3]). The basic idea, lying behind such an attempt, would be that electrons, thermally ejected from potential wells in the reverse direction, should contribute to the current mostly at low fields where the enhancement of the inverse barrier remains sufficiently weak ([4-6]). However, these approaches must be considered cautiously, in so far as authors do not afford a clear vision of the fate of electrons out of wells, and even if the various resulting functions possess an initial plateau. We shall show below that this blurring comes partly from an unfaithfull statement of the manner in which the 
energy of electrons must be accounted for. The third approach consists in a modification of the mood of action of the field, resulting from some kind of interaction between the coulombic wells by themselves. This is classically considered as leading to the so-called Poole regime, in which the permanent current varies with field as $\exp \left(K^{\prime} F\right)$. So that Poole regime can appear as an extension toward the low field range of PF. regime, in so far as interactions between Coulomb potentials can be well stated, and as it can be shown to lead to a new kind of fielddependence. We shall see that authors do not fully agree on the manner in which electrons, on the one hand are freed from donors, and on the other hand take part to the current. The self-consistency of such approaches will not be discussed soundly here, postponing it to a later work. For, presently, we shall simply put into prominence some items in which uncertainties are still remaining. As Poole and PF regimes are commonly used by experimentalists, we wish simply to afford an analysis of the underlying problems, in a more general prospect than that brought by the various available theories considered separately.

Furthermore, it can be recalled besides that the ambiguities emphasized in [1], concerning graphical representations at high fields (PF regime), are still valid in the low field range. Particularly, it was stressed that the whole set of available plots could be regrouped under the general form $\left(\lg j_{0} F^{\mathfrak{m}}, F^{1 / 2}\right)$ when Coulomb potentials are postulated. Let us recall that $m=-1$ stands mainly for PF effect, in the simpler cases of crystalline materials for which mobility $\mu$ can be taken as independent of $F$; $m=0$ is usually associated with Schottky plots, though experimentalists use, commonly, to take it as a PF plot, sometimes without precise justifications ; other positive or negative, fractional, values of $m$ can be chosen following the adopted modelling, in semiconductors [7] as well as in amorphous or polymeric materials $([1,3-5])$.

Having specified and delimited the framework in which to place the present study, we shall build-up two block-diagrams aiming to describe conspicuously the ranges of validity of the various field-enhanced ionization processes, relative to $F$, to the depth $\Phi$ of donors, and to their density $N_{\mathrm{d}}$. Likewise we shall put into prominence the physical limits to be prescribed numerically to these parameters. Furthermore, we shall show that the upper limit of $P F$ regime can be drastically reduced when accounting for tunnelling through the potential barriers of sites [8].

The above considerations indicate therefore that the present paper does not pretend to be an original theoretical contribution. However, we believe it to be of any use to experimentalists, to prick the chart under a synthetic form on the various theoretical approaches, on their uncertainties, and on their practical limits of use.

\section{Basic hypotheses of Poole theory.}

Poole's law was originally set forth to account empirically for permanent currents in Mica [9]. From that time, attemps which have been made to bring theoretical bases to this law are as follows.

(i) The basic phenomenon remains, as in PF regime, a field-induced lowering of potential wells of donors. But instead of being isolated, the wells are supposed to undergo some kind of mutual interaction, which renders electron's thermal freeing more sensitive in the lower field range.

However, three more hypotheses are made, in order to precise further the manner in which interaction takes place.

(ii) Sites, generally taken as Coulomb potentials, are arbitrarily grouped by pairs of nearest neighbours, to which interaction is effectively limited. Pairs are implicitly supposed to be sufficiently apart from one another, to make negligible any external reciprocal influence. Hill [8] considers this hypothesis as necessary. Because, forsaking it would, on the one hand oblige to introduce a screened potential, and on the other hand lead to continuous space change in the medium. The author does not indicate explicitly the way by which screening could occur, but he states that a large array of ionized centres would result in a divergent overall potential. Nevertheless, few authors like Feltz et al. [10] or Bobe and Fritzsch [11] introduce screened potentials empirically. It can be noticed, that despite the pairing of centres, authors use to calculate donor densities $N_{\mathrm{d}}$ as if they were, as a mean, regularly spaced $\left(N_{\mathrm{d}}=a^{-3}, a\right.$ being the mean intersite spacing). Moreover, for the sake of simplicity, pairs are supposed to be aligned along the field direction. As, for pairs pointing toward a perpendicular direction, escaping electrons would experience nearly the potential of a single well [12], and consequently would contribute very little to the current, this is not a very constraining assumption. Finally, to enlarge a little more the outlook on site interactions, it can be mentioned that Dussel and Boër [12] consider pairing only as a matter of simplification. For example, they give an approximate description of mutual modifications of cross-sections of centres, for an ensemble of both attractive and repulsive Coulomb potentials.

(iii) The first site (site 1), situated below with respect to the field direction, is supposed to be filled, while the second site (site 2) is empty. The unusual character of this hypothesis must be commented. Figure 1 shows that, whatever the field $F$, the magnitude of the inner barrier is always lesser than 


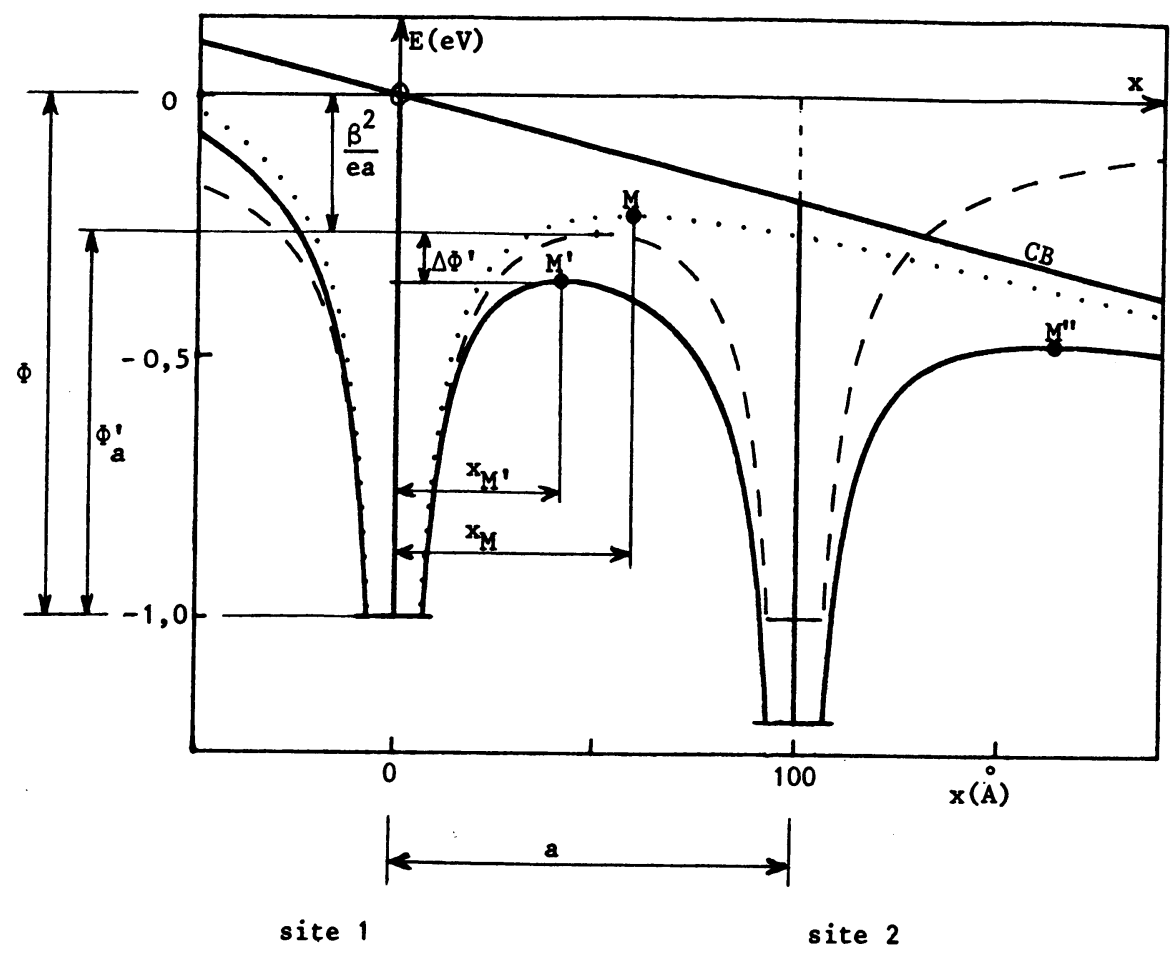

Fig. 1. - Poole regime : potential of an isolated pair of coulombic wells. $\varepsilon=2.2 ; \Phi=1 \mathrm{eV} ; a=100 \AA$; $F=2 \times 10^{7} \mathrm{~V} \cdot \mathrm{m}^{-1} ; F_{\mathrm{t}}=2.610^{7} \mathrm{~V} \cdot \mathrm{m}^{-1}$. (—) $E(x, F) ;(---) E(x, 0) ;(\ldots . . . . . .) E.(x, F)$ for an isolated well.

that of the external barrier. Thus, when $F \neq 0$, site 2 has a greater probability of being occupied than site 1. But, when $F=0$, the probabilities of occupancy are equal for sites 1 and 2 . So that, the present assumption is roughly equivalent to neglect about half of the pairs, if it is admitted that pairs can, as a mean, be occupied by one electron only. It remains none the less, that the necessary enhancement of occupancy of site 2 relative to site 1 , is rather in favour of an immediate re-trapping of electrons liberated from site 1 . But if it were strictly so, no Poole regime could be observed for permanent currents. Thence, this should be the reason why Hill proposed a, hazily defined, low field conduction mechanism through paths formed by limited chains of centres. Moreover in Hill's approach (see Sect. 3), ionization of site 2 over the external potential barrier would be the main source of current at high field strength. Instead, Vollmann [13] considers that electrons freed from site 1 contribute alone the current over the whole field range, apparently without re-trapping in site 2 .

Finally, all authors agree in feeling that Poole regime proceeds from an ionization of the solely occupied site 1 , over the inner potential barrier. Therefore, and despite the quoted discrepancies, it is usually considered that, as long as the inner maximum at $\mathbf{M}^{\prime}$ departs very little from $x_{\mathbf{M}^{\prime}}=$ $a / 2$, the barrier lowering which enhanced ionization proceeds from expresses simply as

$$
\frac{\beta^{2}}{e a}+\frac{1}{2} e a F=\phi^{\prime}+\Delta \phi^{\prime}
$$

with $\beta=\left(e^{3} / \pi \varepsilon \varepsilon_{0}\right)^{1 / 2}$

In this expression, the potential lowering $\phi^{\prime}=$ $\beta^{2} / e a$, due to potential overlap, is distinguished from the field-induced potential lowering $\Delta \phi^{\prime}=$ $\frac{1}{2} e a F$. Consequently, the apparent inner potential barrier height, for wells of depth $\phi$, can be written as

$$
\phi-\phi^{\prime}-\Delta \phi^{\prime}=\phi_{a}^{\prime}-\frac{1}{2} e a F
$$

$\phi_{a}^{\prime}$ appears then as the experimentally observable donor depth. Therefore, in the usual treatments in which the probability of thermal release is approximated by Boltzmann function (see [1]), the steady state current $j_{0}$ can be given its ordinary form

$$
j_{0} \propto \exp \left(\frac{\phi_{a}^{\prime}}{k T}-\frac{e a F}{2 k T}\right) \text {. }
$$

Moreover, as (1b) is meaningless when negative, the quoted model loses any significance when $\phi^{\prime}+$ $\Delta \phi^{\prime}>\phi$. This can occur either when $F$ is large enough, or even at zero field, when $\beta^{2} / e a>\phi$ (strong interaction within pairs at high densities). 
The limits of validity proceeding from both origins will be settled quantitatively in the block-diagram of figure 6. As an additional remark, note that Vollmann considers arbitrarily, as did Hill, that under any field $F$ two sites are said " disconnected ", that is their reciprocal influence is negligible, when their distance $a$ is larger than

$$
a_{\min }=2 x_{\mathrm{M}}(F)=\frac{\beta}{e} F^{-1 / 2}
$$

$x_{\mathrm{M}}$ being the potential maximum for a single well (see Fig. 1). Therefore, for a given $a$, sites disconnected at low fields can become connected at higher fields. As will be seen below, the notion of connection, of minor importance in Hill's statements, becomes fundamental in the approaches of Vollmann, and of Dussel and Boër. Moreover, it will appear that no straightforward relation can be settled between connection and Poole or PF laws.

\section{Transition from Poole regime to PF regime.}

The preceding considerations having shown the very akin nature of the available interpretations of Poole and $\mathrm{PF}$ regimes, it would remain to see how these regimes become successively dominant, when $F$ is increased over an extended interval. However, as this depends on the hypotheses subtending the models, an outline is needed of the manner in which authors do consider the problem.

3.1 TRANSition FOllowing VollmanN. - In a one-dimensional calculation, the potential of a pair under an applied field, is given classically by

$$
E(x, F)=-\frac{e^{2}}{4 \pi \varepsilon \varepsilon_{0}}\left(\frac{1}{|x|}+\frac{1}{|a-x|}\right)-e F x
$$

when the origin is taken at the centre of site 1 .

Differentiating and then equating to zero would give two real maxima $x_{\mathrm{M}^{\prime}}$ and $x_{\mathrm{M}^{\prime \prime}}$ (see Fig. 1) as solutions of a fourthdegree polynomial. A tractable derivation can be found in [14]. Instead, Vollmann choose to perform an iterated determination of, only, the inner maximum. The author introduces into this polynomial the distance $x_{\mathrm{M}}$ of the turningpoint, for a single well. The iterative procedure can then be implemented in two ways, corresponding respectively to low fields for which $x_{\mathrm{M}}>\frac{a}{2}$, and to high fields where $x_{\mathrm{M}}<\frac{a}{2}$.

So, in the low field range, $x_{\mathrm{M}^{\prime}}$ approximates to

$$
x_{\mathrm{M}^{\prime}} \sim \frac{a}{2}\left(1-\frac{1}{4}\left(\frac{a}{2 x_{\mathrm{M}}}\right)^{2}\right)=\frac{a}{2}\left(1-\frac{1}{4} \frac{F}{F_{\mathrm{t}}}\right)
$$

if one introduces the parameter

$$
F_{\mathrm{t}}=\left(\frac{\beta}{e a}\right)^{2}=N_{\mathrm{d}}^{2 / 3}\left(\frac{\beta}{e}\right)^{2} .
$$

In this relationship, the corrective factor is limited upward by $1 / 4$, as $a / 2 x_{\mathrm{M}}<1$.

Vollmann determines then the field enhanced permanent current, in a model similar to Simmons' [15]

$$
\begin{aligned}
j_{0}=e \mu\left(N_{\mathrm{c}} N_{\mathrm{d}}\right)^{1 / 2} F \exp \left(-\frac{\phi_{a}^{\prime}}{2 k T}\right) \times \\
\times \exp \left(\frac{\alpha^{\prime}}{2}\left(1-\frac{f}{4}\right)\right)
\end{aligned}
$$

with $\alpha^{\prime}=\frac{1}{2} \frac{e a F}{k T}$, and $f=F / F_{\mathrm{t}}$.

Thus, a Poole's law, slightly damped by the term $1-f / 4$, is found in the lower field range.

In the high field range where $x_{\mathrm{M}}<\frac{a}{2}$, an alike iteration procedure, leads however to another expression for $x_{\mathrm{M}^{\prime}}$

$$
x_{\mathrm{M}^{\prime}} \sim x_{\mathrm{M}}\left(1-\frac{1}{8 f}\right) .
$$

Consequently, the permanent current becomes of a PF-type, slightly augmented by the correction factor $1+1 / 8 f$ with $f>1$

$$
\begin{aligned}
j_{0}=e \mu\left(N_{\mathrm{c}} N_{\mathrm{d}}\right)^{1 / 2} F \exp \left(-\frac{\phi-\phi^{\prime} / 4}{2 k T}\right) \times \\
\times \exp \left(\frac{\beta \sqrt{F}}{2 k T}\left(1+\frac{1}{8 f}\right)\right) .
\end{aligned}
$$

At the outset, the iteration process makes $F_{\mathrm{t}}$ to appear as a transition field, from Poole regime to PF regime. But it is essential at this point to emphasize that, in Vollmann's model, Poole and PF laws arise uniquely as a consequence of thermal release of electrons over the inner barrier. So that the transition from the former law to the latter can be explained by the fact that, at higher fields, the remainder of the barrier is less sensitive to distorsion by the field.

To take a limiting example of possible magnitudes of the transition field, $F_{\mathrm{t}} \sim 10^{7} \mathrm{~V} \cdot \mathrm{m}^{-1}$ with $N_{\mathrm{d}} \sim$ $10^{17} \mathrm{~cm}^{-3}$ and $\varepsilon=2.2$. So, for very high donor densities, as can be found in amorphous materials, Poole regime is likely to extend up to pre-breakdown fields.

\subsection{TRANSITION FIELD DEDUCED FROM HILl's AP-} PROACH. - In Hill's approach transition is also present, but indirectly as it does not constitute a fundamental item, Poole and PF regimes receiving here different interpretations, as seen above. In the low field range where electrons are supposed to be excited over the inner potential barrier, the author considers that $x_{\mathrm{M}^{\prime}}$ departs very little from $a / 2$. Consequently, equation (2) is found, in a one-dimensional approach. The range of low fields is 
delimited by writing $x_{\mathrm{M}^{\prime}}=\frac{a}{2}-\delta x$, so that $\frac{\delta x}{a / 2}=$ $\frac{e^{2} a^{2}}{4 \beta^{2}} F=\frac{1}{4} f<1$. This condition (Eq. (23) of Hill) re-written in terms of $f$ (see Eq. (5b)), states then that Poole's law can be effective only when $f<4$. Equivalently this condition would reduce to $f<1$, if the limiting field of Hill was taken four times larger than Vollmann's transition field. Besides, Hill does . not attempt to evaluate a correction factor.

For higher fields, in contrast to Vollmann's treatment, Hill contrives a model of ionization of site 2 by way of two competing processes : thermal freeing over the external barrier ; and tunnelling through it, with a possibility of thermally assisted tunnelling in the medium field range. In this model, an electron in site 2 is considered as experiencing a potential nearly equivalent to that of a doubly-charged, single coulombic well, located midway the sites in a pair. We showed elsewhere [14] this is only a crude approximation.

3.3 Transition fOllowing Dussel AND BoËr. - In Dussel and Boër approach, the field enhanced ionization seems to be dealt with the inner potential barrier only, as in Vollmann's model. But in contrast with all other authors who use to derive the probability of thermal ionization only, they tried to write down properly the conditions for the steady state density of occupation of donor sites, for many valued sets of parameters $N_{\mathrm{d}}, F$ and $T$. To do so, they proposed separate evaluations of the capture cross-section and of the probability of thermal ionization, with or without a field applied. Let us recall that, as did other authors, Dussel and Boër consider only paired wells aligned along the field direction, and that pairing is considered as a particular example of mutual interactions between potential wells, either attractive or repulsive. The authors' developments can then be summarized as follows.

3.3.1 No field applied. - Figure 2 inspired from the authors' presentation, is a drawing, with no field applied, of their attainments about capture crosssections. $2 r_{\mathrm{A}}(0)$ is the diameter of an isolated coulombic well truncated at a depth $2 k T$. Such truncation delimits an equipotential surface called critical surface, on which electrons get an equal probability of being either freed or recaptured. Sites are considered as becoming disconnected, whenever the inner potential maximum $E\left(x_{\mathrm{M}^{\prime}}\right) \leqq 2 k T$. Figure 2 shows clearly that this happens for an inter-site distance.

$$
a=4 r_{\mathrm{A}}(0) \text {. }
$$

This equation, contrary to equation ( $3 a$ ), gives a finite minimum density for disconnection when $F=0$. Then, the actual profile of capture critical surfaces are as indicated in figure 2. As an example, Dussel and Boër calculated that a partial potential overlap can arise, in a low temperature range, for densities as smaller as $10^{15} \mathrm{~cm}^{-3}$, with no field applied $\left(a \sim 10^{3} \AA\right)$.

When sites are said disconnected, this does not imply that their critical surfaces remain undistorted.
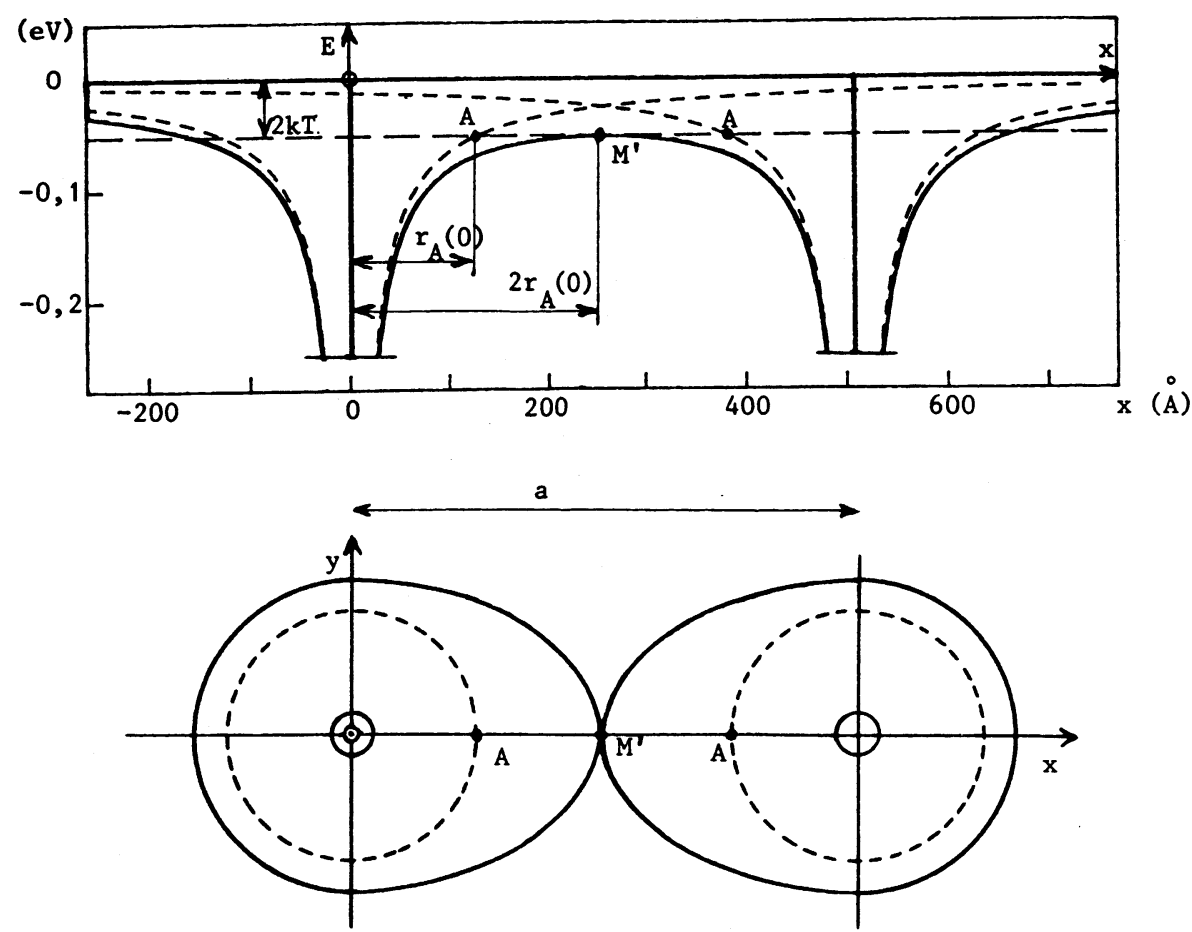

Fig. 2. - Limiting case of site connection without applied field. $N_{\mathrm{d}}=7.6 \times 10^{15} \mathrm{~cm}^{-3} ; \varepsilon=2.2 ; T=300 \mathrm{~K}$. 
But simply that, on the two sides of the transition occurring as $a$ is varied, their influence on probabilities of occupation is effective or not.

We deduced from these results a particular temperature effect. The potential lowering in zero-field condition being determined through equation (4) as equal to $\beta^{2} / e a$, disconnection would occur, at point $\mathbf{M}^{\prime}$, whenever

$$
\frac{\beta^{2}}{e a}=2 k T .
$$

This equation means that, for any given (and slightly temperature dependent) density of sites $N_{\mathrm{d}}$, there would exist a transition temperature :

$$
T_{\mathrm{t}}=\frac{\beta^{2}}{2 e k} N_{\mathrm{d}}^{1 / 3}
$$

such that for $T<T_{t}$, centres should be connected, whilst for $T>T_{\mathrm{t}}$ they should be disconnected. But, as shown by figure 3 , this transition could appear at realistic temperatures only for rather small densities. Point $\mathbf{P}$ in the figure corresponds to an estimation of density, calculated by Dussel and Boër, as equal to $N_{\mathrm{d}}=1.7 \times 10^{17} \mathrm{~cm}^{-3}$, with $\varepsilon=10$ and for a temperature of $200 \mathrm{~K}$. In accordance with our point of view, this temperature should be the transition temperature $T_{\mathrm{t}}$.

3.3.2 Cross-section modifications by field. - Then the field influence is represented in figure 4 . If the inter-site distance is so small as to make zero-field connection (Fig. 4a), the representation under a field sufficiently weak is that of figure $4 \mathrm{~b}$. Fieldenhanced ionization is then governed by thermal freeing over the saddle point, giving rise to a Poole mechanism. Raising the field brings $\mathbf{M}^{\prime}$ closer to the horizontal line (P) lying $2 k T$ below $\mathrm{M}^{\prime \prime}$, and sites are disconnected when $M^{\prime}$ reaches $(P)$. This occurs for a transition field, that we keep on labelling $F_{t}$, as in Vollmann's approach. Hence, for any field $F>F_{\mathrm{t}}$ (Fig. 4c) a PF regime sets in. Because then, the residual barrier height that electrons of sites 1 have to overcome is limited by line (P), and practically not by the reciprocal deformations of paired potentials. So, a high field PF regime results, originating, as in Vollmann's treatment, and despite a different approach, in ionization of site 1.

Distinguishing between Poole and PF regimes can cease to be an actual problem, when screened potentials are worked out. For then, potentials could become so steep that site connection should be no more available. Hence, a unique mathematical treatment would give access to field-enhanced ionization. However; this would need to contrive suitable screening models. This will not be undertaken here, delaying a survey of this matter to a future paper. The only thing to be made here is simply to mention a theoretical attempt of Feltz et al. [10], and to examine the behaviour resulting from an empirical treatment by Fritzsch and Bobe [16].

3.3.3 Graphical approaches of Fritzsch and Bobe. Fritzsch and Bobe, and Bobe and Fritzsch [11], supposing as usual, that Boltzmann function is a suitable approximation of Fermi-Dirac statistics, put forward, with the help of both graphical representations of experimental data and of computer simulations, the close affinity liable to exist between the screened-coulombic-well model and the coulombicpaired-well model.

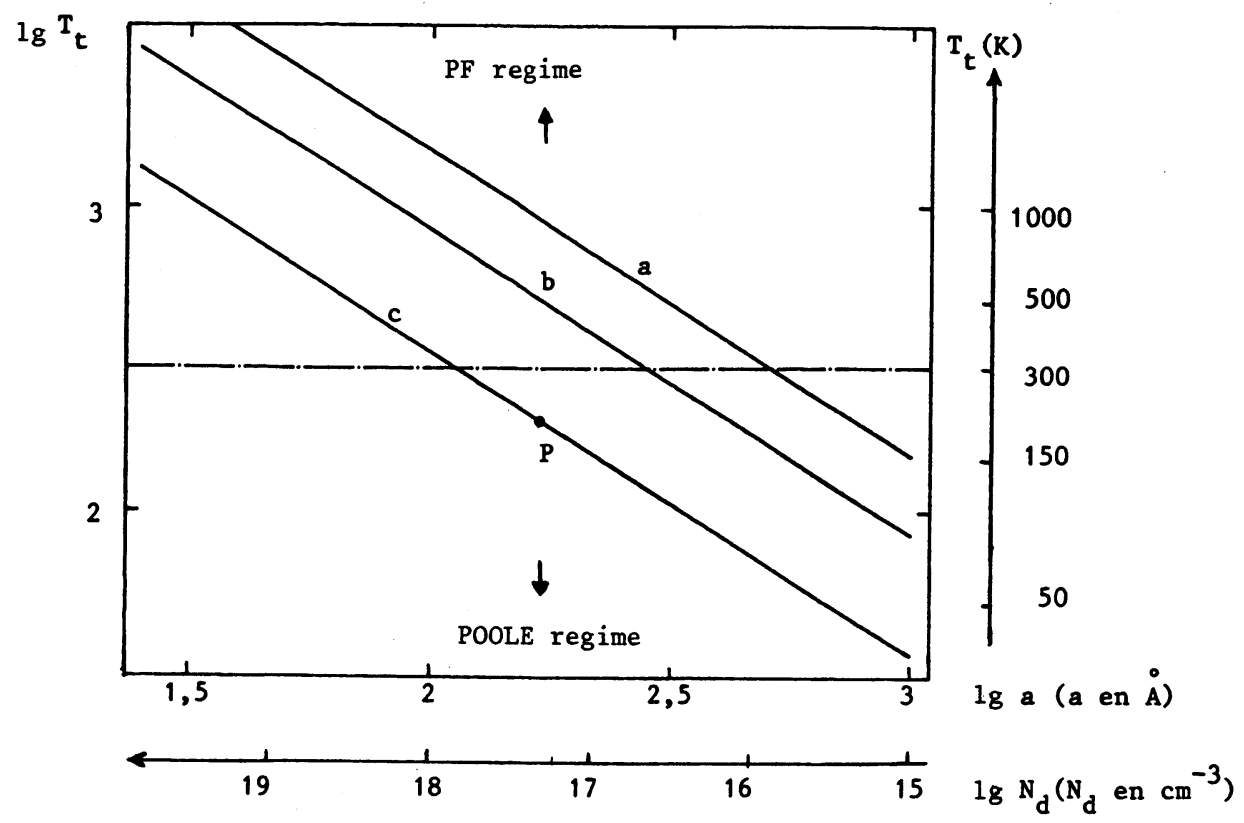

Fig. 3. - Influence of site density $N_{\mathrm{d}}$ on temperature $T_{\mathrm{t}}$ of transition from Poole to PF regimes. a) $\varepsilon=$ 2.2 ; b) $\varepsilon=4$; c) $\varepsilon=10$. 


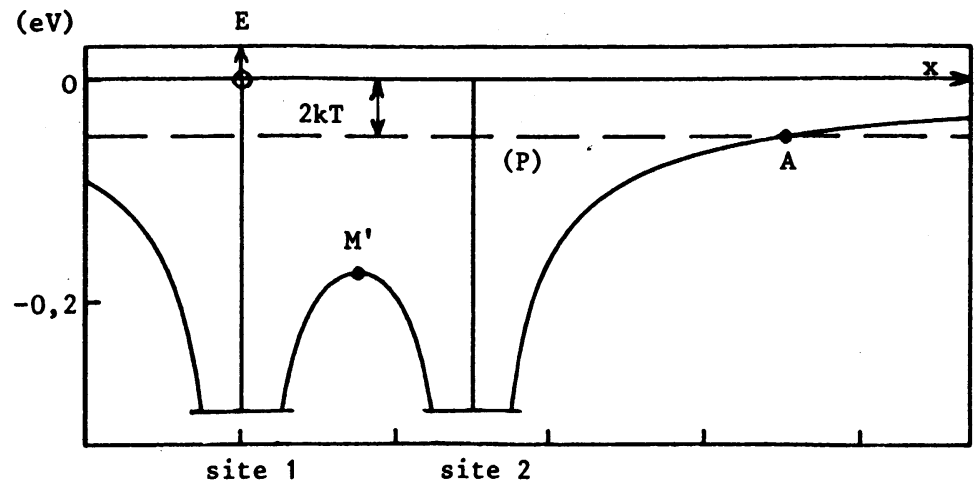

a)

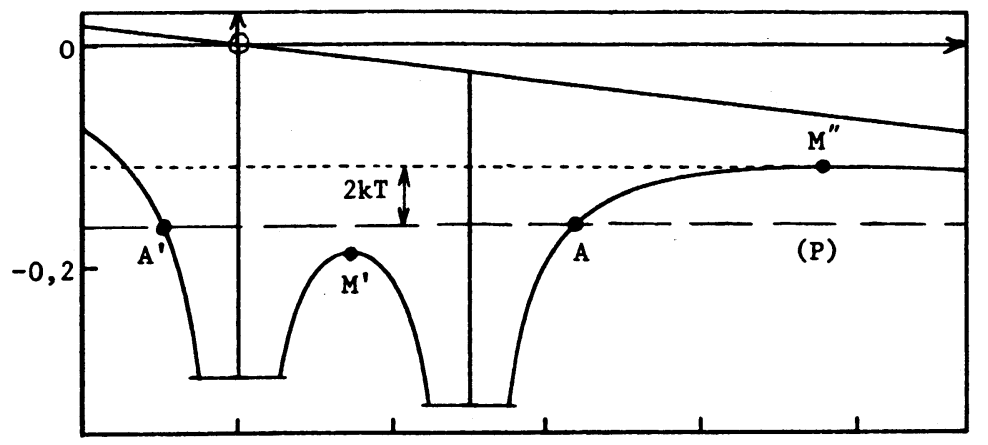

b)

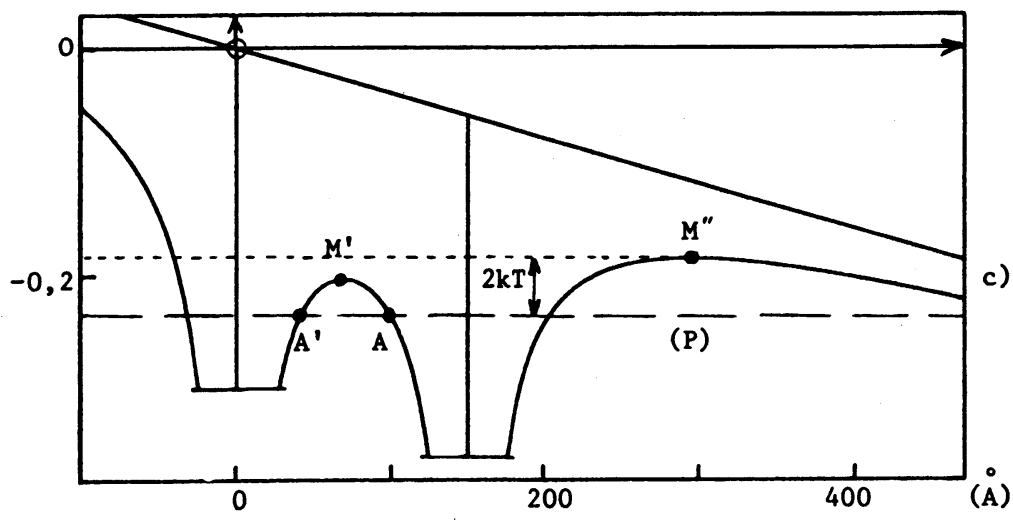

Fig. 4. - (Following Dussel and Boër [9]) Connection-disconnection of paired sites by the field, with $N_{\mathrm{d}}=$ $3 \times 10^{17} \mathrm{~cm}^{-3}, \varepsilon=2.2$ and $T=300 \mathrm{~K}$. Connected centres : a) with $F=0 ;$ b) with $F<F_{\mathrm{t}}$. Disconnected centres : c) with $F>F_{\mathrm{t}}$.

In the latter case, authors determine numerically [16] the inner barrier lowering $\Delta \Phi(F)$, responsible for field enhanced ionization. So, they establish that $\Delta \Phi$ may be written empirically under the form :

$$
\Delta \Phi(F)=K(\varepsilon, a) F^{\mathfrak{n}(F, \varepsilon, a)} .
$$

Variations of $\boldsymbol{n}$ in terms of $a$, and for constant field, are represented in figure 5 . This figure indicates that $n$ ranges within 0.5 and 1 , as for the multipolar potentials or Arnett and Klein [17].

In the case of the screened-coulombic model [11], graphical data representation allows them to show that $\Delta \Phi(F)$ can be expressed alike by :

$$
\Delta \Phi(F)=K\left(\varepsilon, r_{0}\right) F^{\mathfrak{n}\left(F, \varepsilon, r_{0}\right)} .
$$

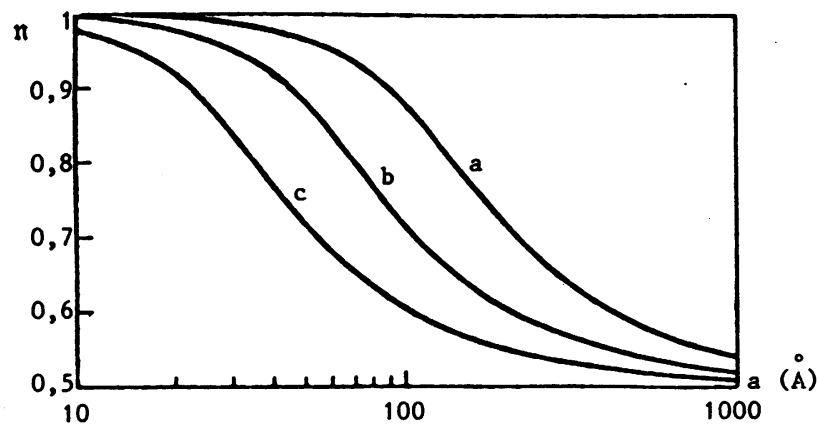

Fig. 5. - (Following Fritzsch and Bobe [12]) Variations of slope $n$ with distance $a$, for a coulombic well pair. $F=6 \times 10^{7}$ V.m ${ }^{-1}$; a) $\varepsilon=1$; b) $\varepsilon=4$; c) $\varepsilon=16$ 
In this equation, the screening distance $r_{0}$ is taken as proportional to $n^{-s} ; n$ being the free-carrier density, and not the density of donor sites as in the treatment of Feltz et al. [10] ; $s$ being a suitable parameter.

Fritzsch and Bobe propose for permanent current, the empirical form :

$$
j_{0} \propto \exp \left(-\frac{\Phi-\Delta \Phi(F)}{K_{1} T^{3 / 2}}\right)
$$

where $K_{1}$ is an appropriate constant. They verified that this formulation provides a convenient fitting of data representation of currents $j_{0}(V)$, in a $\left(\lg j_{0} / V\right.$, $\lg V$ ) plot.

Though empirical, the authors' approach appears as very interesting, in so far as it tries to bring together two apparently irrelevant methods of treatment of interactions.

\section{Block diagram of Poole and PF regimes.}

Bearing in mind the clear distinction made above, between Poole and PF regimes, together with the notion of saturation developed in [1], it becomes easy to build up the block diagram of figure 6 . Coordinates are chosen in order to emphasize sharply the role played by the sensitive parameters of the system. However, vertical axis is graduated in $F$, so that the actual shape of curves (s), (t) and $\left(t_{s}\right)$ depends on the peculiar «generalized» $P F$ regime under consideration (potentials either coulombic or non-coulombic). Then some subsidiary lack of universality results from.

Vollman's transition field $F_{\mathrm{t}}$ generates a cylindrical surface (T), which axis parallels $O \Phi$, and leaning on curve $(\mathrm{t})$ defined by equation $F_{\mathrm{t}}=(\beta / e a)^{2}$. Thus, this sheet divides space in two regions, the lower

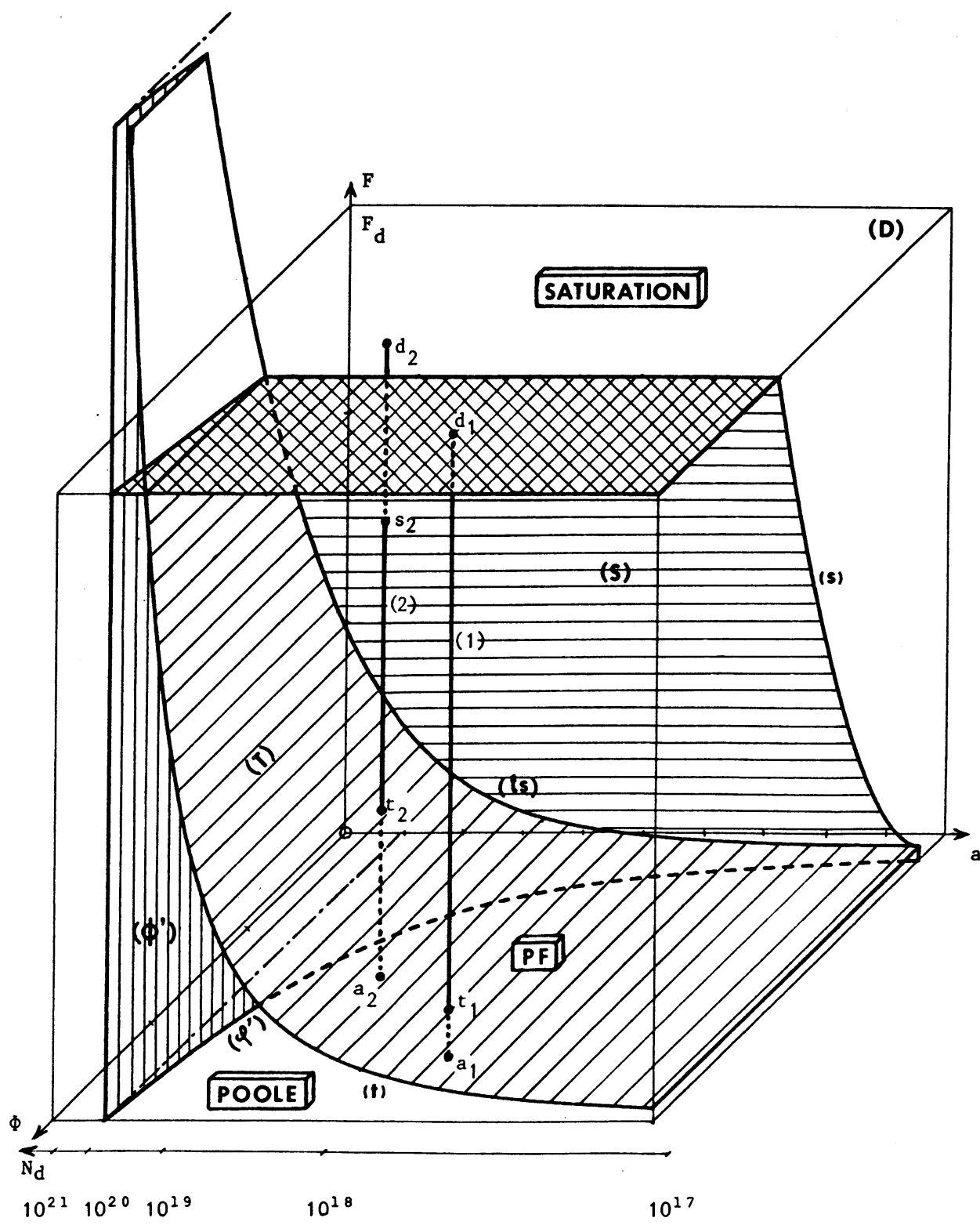

Fig. 6. - Synthetic representation in $\Phi, a$ and $F$, showing the respective ranges of Poole and PF regimes, and including saturation. Variable ranges : $0 \leqq \Phi \leqq 1: 5 \mathrm{eV} ; 0 \leqq a \leqq 200 \AA ; 0 \leqq F \leqq 3 \times 10^{8} \mathrm{~V} \cdot \mathrm{m}^{-1} \cdot \varepsilon=2.2$. 
corresponding to Poole regime, and the higher to PF regime. Its actual position depends only upon dielectric constant as $F_{\mathrm{t}} \propto \varepsilon^{-1}$. Note that the transition field of Vollmann is used only as a matter of convenience, though it leads to a marked discontinuity of Poole and PF curves at this point, contrarily to the transition field deduced from Hill's treatment, for which continuity is achieved. Therefore, (T) must be considered as a lower limit of validity of Poole regime.

Saturation field $F_{\mathrm{s}}$, previously defined in [1], generates for its part a cylindrical sheet (S), of axis $\mathrm{O} a$, and leaning on curve (s) of equation $F_{\mathrm{s}}=$ $(\Phi / \beta)^{2}$. The actual position of this surface depends only on $\varepsilon$, for $F_{\mathrm{s}} \propto \varepsilon$. Sheets (T) and (S) intersect on curve $\left(\mathrm{t}_{\mathrm{s}}\right)$, along which $F_{\mathrm{t}}=F_{\mathrm{s}}$. Surface (S) delimits two regions in space, which rear part is the domain where PF regime is saturated. However, it must be reminded that $(S)$ represents only the locus of the maximum $F$ magnitudes for which saturation is effective. But actually, saturation settlement is not a so clear-cut phenomenon and, as was shown in [1], sets in progressively from $F$ values sometimes far below $F_{\mathrm{s}}$. Under such a reservation, the fore part of space relative to $(\mathrm{S})$ is the domain of existence of PF regime.

Likewise curve $\left(\varphi^{\prime}\right)$, defined by equation $\Phi^{\prime}=$ $\beta^{2} / e a$, generates a cylindrical surface $\left(\Phi^{\prime}\right)$, of axis parallel to $\mathrm{O} F$. This surface represents the locus in space along which $\Phi_{a}^{\prime}=0$. As $\Phi_{a}^{\prime}=\Phi-\beta^{2} / e a$ holds in general conditions $\left(\Phi^{\prime}\right)$ divides space into two regions. The part of the forward region which, in addition, is limited upwards by sheet $(\mathrm{T})$, delimits the volume of Poole regime validity. This volume is also that where site 1 remains defined, and at least partially occupied under zero-field condition. Rear part is the region in space where sites 1 and 2 (Sect. 2 above) cease to be defined, because the inner potential maximum with no field applied lies then below their ground states. Consequently these sites are entirely empty. More fundamentally it can be said that the paired-well entity as a whole has then lost any physical meaning. Hence, in the portion of space delimited by cylinder $\left(\Phi^{\prime}\right)$ and by planes $F \mathrm{O} a$ and $F \mathrm{O} \Phi$, neither Poole nor PF regimes can exist. As in this region donors are necessarily empty, a saturated (ohmic) conduction would only work, at least for fields lesser than avalanche threshold.

Plane (D) parallel to plane $\Phi \mathrm{O} a$, and of equation $F=F_{\mathrm{d}}, F_{\mathrm{d}}$ being the breakdown field, gives the uppermost boundary of experimentally attainable points in space. Moreover, points lying between plane $F O \Phi$ and a plane parallel to it, and of equation $a_{\text {mini }}$, have to be excluded from regions physically accessible. Because, in the highest densities that can be anticipated, Coulomb sites are necessarily many $\AA$ apart. In figure $6, \Phi$ was given a maximum value of $1.5 \mathrm{eV}$. This implies a density of sites amounting to $1.9 \times 10^{20} \mathrm{~cm}^{-3}$, a value rather high even in amorphous materials, and despite its accordance with values considered by Hill.

Finally the available volume is bounded by plane (D), vertical cylinder $\left(\Phi^{\prime}\right)$, and horizontal cylinder (S), with in addition internal boundary $(T)$. Two exemplary types of field action can then be described. (i) When, for the material under investigation, the equilibrium condition $(F=0)$ is given by a point like $a_{1}$, any field application would make a sliding up of the representative point, along the segment $a_{1} d_{1}$. Then, Poole regime is present on the reduced interval $a_{1} t_{1}$ only, while $P F$ regime is running up to $d_{1}$ (breakdown field). (ii) When the equilibrium condition is represented by a point like $a_{2}$, the segment $a_{2} d_{2}$ is described upward when field is growing. But now, the interval over which Poole regime sets in, is very extended, whilst $P F$ regime appears only on the short interval $t_{2} s_{2}$. Above $\mathrm{s}_{2} \mathrm{~d}_{2}$, a saturated regime sets in until breakdown occurs.

\section{Tunnel effect predominance below saturation.}

5.1 TheORETiCAL STATEMENTS. - The preceeding block diagram must be modified, if an electron tunnelling, through the field reduced potential barriers, is taken under consideration. This was worked out by Hill [8] for any field strength. In order to give the simplest presentation of Hill's calculations, we restrict the present account to tunnelling through the external barrier at high field strengths only (PF regime).

Hill considers, as shown by figure 7 , three possibilities for electrons to escape from isolated wells.

(i) At temperatures and fields sufficiently high, direct tunnelling is predominant (path 1 of Fig. 7a). By high field, it is intended a field higher than a field for which the probabilities of ionization by tunnel effect and by PF effect are equal. (ii) At sufficiently high temperatures, and for moderate fields, thermal ionization prevails (path 2). (iii) For intermediate cases, a thermally assisted tunnelling through the field-reduced barrier can be considered as the main ionization mechanism (path 3).

Among these possibilities, the third seems to be the most convenient for a great deal of common dielectric studies. In this case, Hill considers (Fig. 7c) that an electron is able to pass through the barrier after being excited to a level $E_{0}$, distant of an amount $\Phi_{0}$ from the fundamental level $E_{\mathrm{d}}$ of the site. $\Phi_{0}$ is limited by the condition : $\Phi_{0} \leqq \Phi-$ $\Delta \Phi, \Phi$ being the site depth, $\Delta \Phi$ the PF barrier reduction. This case is referred to hereafter as PF assisted tunnelling (PFAT). 


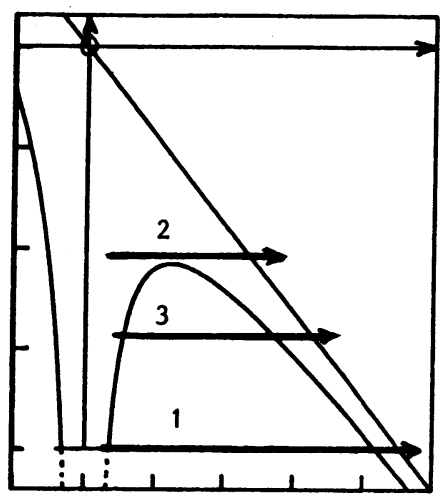

a)

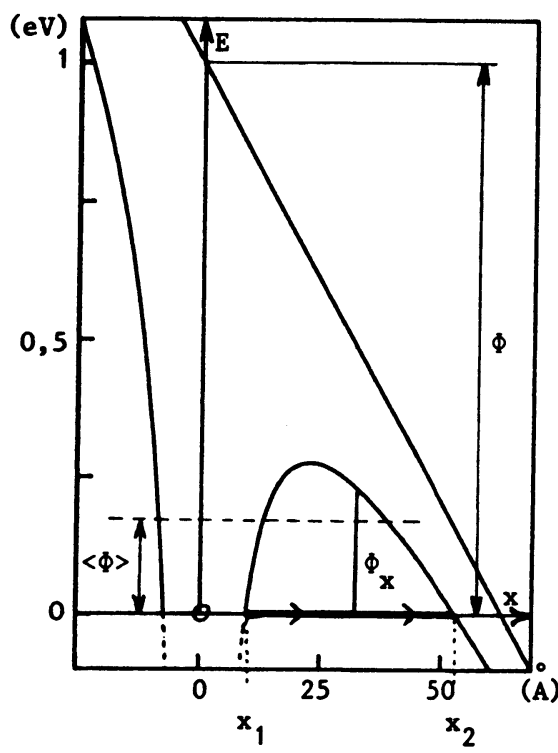

b)
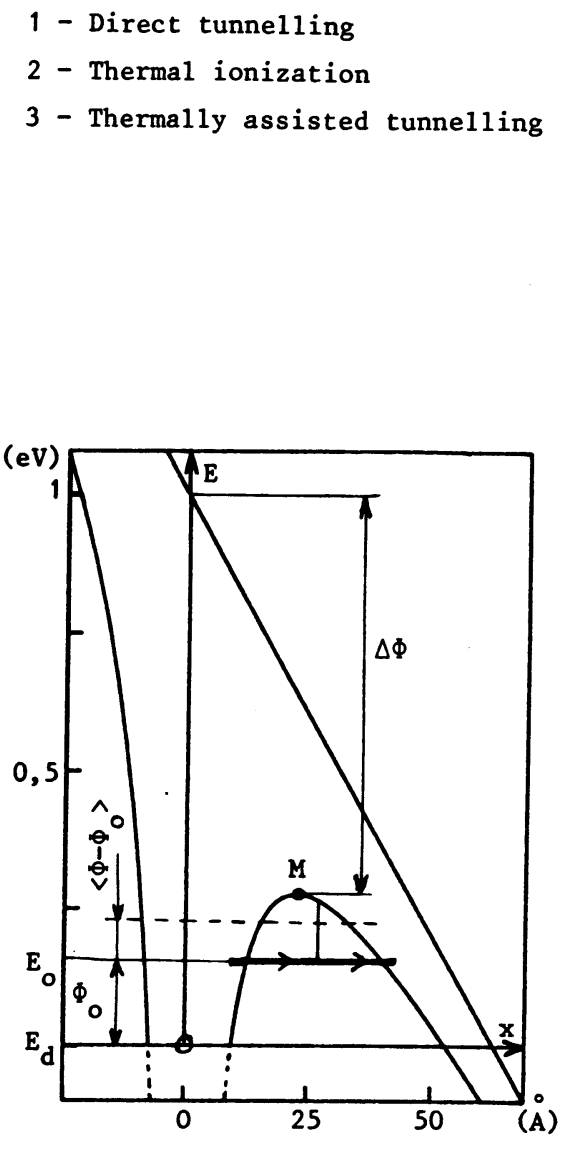

c)

Fig. 7. - Barrier profiles for tunnelling in Hill's model [3]. a) The various tunnelling processes. b) Direct tunnelling $\varepsilon=2.2 ; m^{*}=0.2 m_{\mathrm{c}} ; \Phi=1 \mathrm{eV} ; F=2 \times 10^{8} \mathrm{~V} \cdot \mathrm{m}^{-1} ; T \leqq 5 \mathrm{~K}$ so that $\left(\Phi_{0}\right)_{\mathrm{M}} \leqq 10^{-2} \mathrm{eV}$ be negligible with respect to $\Delta \Phi=0.72 \mathrm{eV}$. c) Thermally assisted tunnelling, in the same conditions, with $T=300 \mathrm{~K} ;\left(\Phi_{0}\right)_{\mathrm{M}}=0.16 \mathrm{eV}$ (see Fig. 8, curve (b)).

The barrier transparency $\mathfrak{C}(E)$ is then roughly determined, by :

$$
\begin{aligned}
\mathfrak{C}(E)=\exp \left(-A\left\langle\Phi-\Phi_{0}\right\rangle^{1 / 2}\right) & \times \\
& \times \exp \left(-B\left(E_{\mathrm{F}}-E_{x}\right)\right) .
\end{aligned}
$$

In this equation : $E_{\mathrm{F}}$ is the Fermi energy ; $E_{x}$ is the energy component along the field direction of an electron in the well ;

$$
A=2 \frac{\Delta x}{\hbar}\left(2 m^{*}\right)^{1 / 2} ; B=A / 2\left\langle\Phi-\Phi_{0}\right\rangle^{1 / 2} ;
$$

$\Delta x=x_{2}-x_{1}$ is the barrier width ; and $\left\langle\Phi-\Phi_{0}\right\rangle$ is the mean barrier height, along the field direction, for an electron raised at level $E_{0}$. Figure $7 \mathrm{~b}$ is the particular case where $\Phi_{0} \sim 0$, related to a pure tunnelling.
Then Hill determines after few further simplifications, an expression for the current valuable when a narrow impurity band is stated. Parameters in $\mathfrak{C}(E)$ can then be written :

$$
\begin{aligned}
A\left\langle\Phi-\Phi_{0}\right\rangle^{1 / 2} & =\frac{\sqrt{2}}{\left(\Phi-\Phi_{0}\right)^{1 / 2}} \frac{\left(2 m^{*}\right)^{1 / 2}}{\hbar e} \times \\
& \times \frac{\beta^{2} \cdot \lambda_{a}}{f_{a}}\left(1-\frac{f_{a}}{2 \lambda_{a}} \operatorname{Ln} \frac{1+\lambda_{a}}{1-\lambda_{a}}\right)^{1 / 2} ; \\
\lambda_{a} & =\left(1-f_{a}\right)^{1 / 2} ; \quad f_{a}=\frac{F}{F_{\mathrm{s} a}}
\end{aligned}
$$

and

$$
F_{s a}=\left(\frac{\Phi-\Phi_{0}}{\beta}\right)^{2}
$$


Notation $F_{\mathrm{s} a}$ is introduced as this ratio appears as very close in form to the expression for the field of saturation $F_{\mathrm{s}}$, and as $F_{\mathrm{s} a} \rightarrow F_{\mathrm{s}}$ when $F$ becomes very large. The above equations simplify when $\lambda_{a} \ll 1$. Actually this inequality is not a too restraining condition for, the error incurred in it reaches $10 \%$ only for $\lambda_{a}=0.5$. $\left(f_{a}=0.75\right)$. Then :

$$
\begin{aligned}
A\left\langle\Phi-\Phi_{0}\right\rangle^{1 / 2} \sim \frac{2}{\hbar} \frac{\left(2 m^{*}\right)^{1 / 2}}{e F}( & \left.\Phi-\Phi_{0}-\Delta \Phi\right) \times \\
& \times(\Phi-\Delta \Phi) / \Phi_{0}^{1 / 2} .
\end{aligned}
$$

If, as usual in all works published up to now on PF effect, Fermi-Dirac statistics is approximated by Boltzmann function, the overall probability of ionization, together with the permanent current $j_{0}$, are proportional to :

$$
\exp \left(-A\left\langle\Phi-\Phi_{0}\right\rangle^{1 / 2}-\frac{\Phi_{0}}{k T}\right) .
$$

Expressing that $j_{0}$ is going through a maximum when $\Phi$ amounts to its maximum value $\left(\Phi_{0}\right)_{\mathrm{M}}$, and if it is assumed that the main current component proceeds then from thermally assisted tunnelling, $j_{0}$ expresses as :

$$
\begin{aligned}
& j_{0}=K \exp \left(-\frac{2 K_{0} T^{2 / 3}}{k T}\left(\frac{\Phi}{\sqrt{F}}-\beta\right)^{4 / 3} \times\right. \\
&\left.\times\left(1-\frac{K_{0} T^{2 / 3}}{2 \sqrt{F}}\left(\frac{\Phi}{\sqrt{F}}-\beta\right)^{1 / 3}\right)\right) .
\end{aligned}
$$

Following O'Dwyer [18] this form would fit rather well with experimental data, for high field strengths and moderate or high temperatures.

To illustrate what happens when $F$ is varied, we have represented in figure 8 the respective variations of $\Phi-\Delta \Phi$ and of $\left(\Phi_{0}\right)_{M}$. This drawing, not previously made by Hill, afforded us a quantitative estimation of the involved parameters. It allows, moreover, to show that, depending heavily upon temperature, there exists a transition field $F_{a}$, from jumping over the barrier to tunnelling, for which $\left(\Phi_{0}\right)_{\mathrm{M}}=\Phi-\Delta \Phi$. Therefore, for fields $F>F_{a}$ the PFAT regime is dominant, while a PF regime is working for $F<F_{a}$ (and $F>F_{\mathrm{t}}$ ).

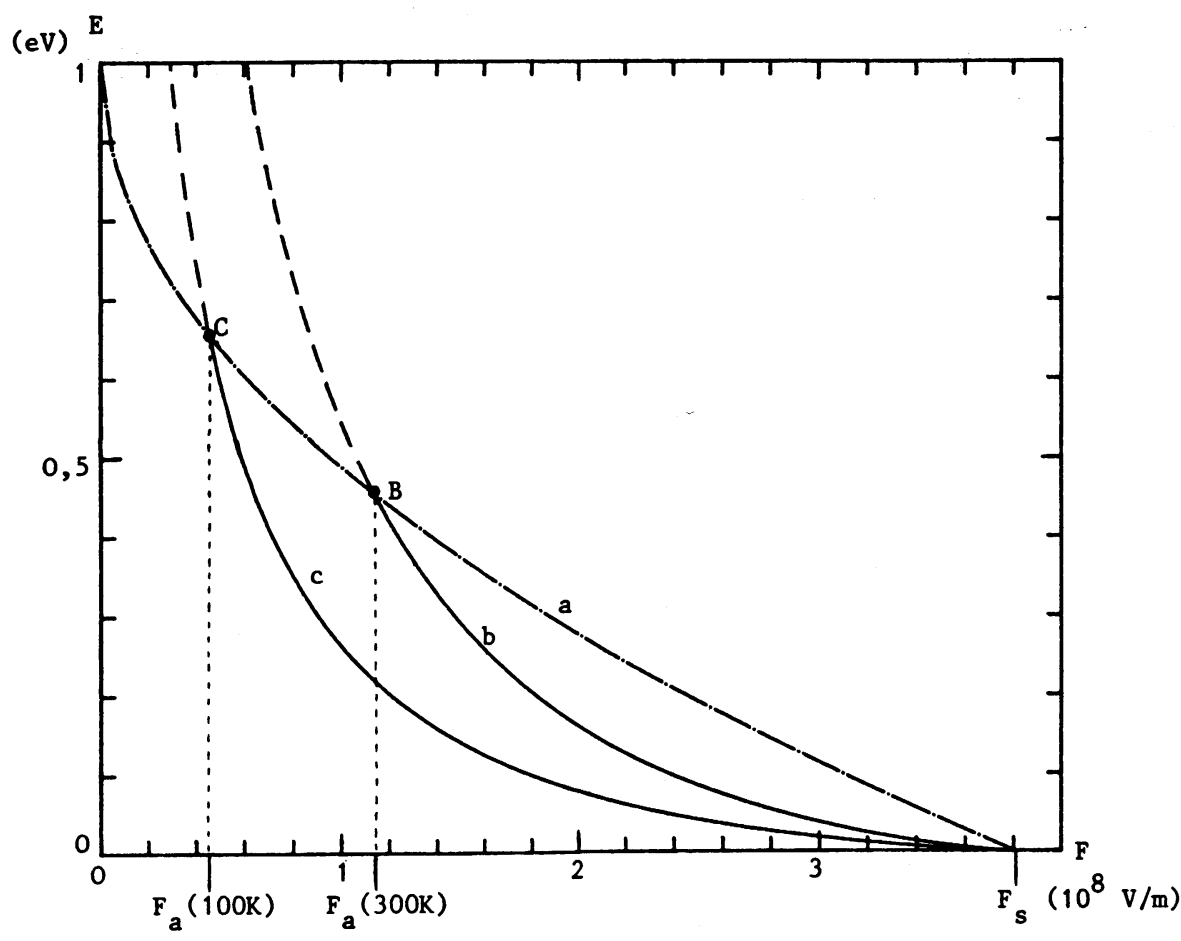

Fig. 8. - Hill's model of PF assisted tunnelling (PFAT). Variations versus $F$ of : (a) $\Phi-\Delta \Phi$; (b) ( $\left.\Phi_{0}\right)_{M}$ at $T=300 \mathrm{~K} ;$ (c) $\left(\Phi_{0}\right)_{\mathrm{M}}$ at $100 \mathrm{~K} . \varepsilon=2.2 ; m^{*}=0.2 m_{\mathrm{e}} ; \Phi=1 \mathrm{eV}$. Dashed parts of curves (b) and (c) correspond to a regime where tunnelling cannot work.

5.2 BLOCK DIAGRAM INVOLVING TUNNELLING. Figure 9 gives a representation of the surfaces separating regions in space where PF regime and PFAT regime are successively predominant as $F$ is increased. These surfaces are cylindrical sheets, of axis $\mathrm{O} a$, generated by curves (n) corresponding to $\left(\Phi_{0}\right)_{\mathrm{M}}$. Two sheets $(\mathrm{N})$ and $\left(\mathrm{N}^{\prime}\right)$ are drawn, the lower for $T=200 \mathrm{~K}$, the upper for $T=500 \mathrm{~K}$. Curves $\left(t_{n}\right)$ and $\left(t_{n}^{\prime}\right)$ are the intersections of $(\mathrm{N})$ and $\left(\mathrm{N}^{\prime}\right)$ with $(\mathrm{T})$. Sheets are not protracted 


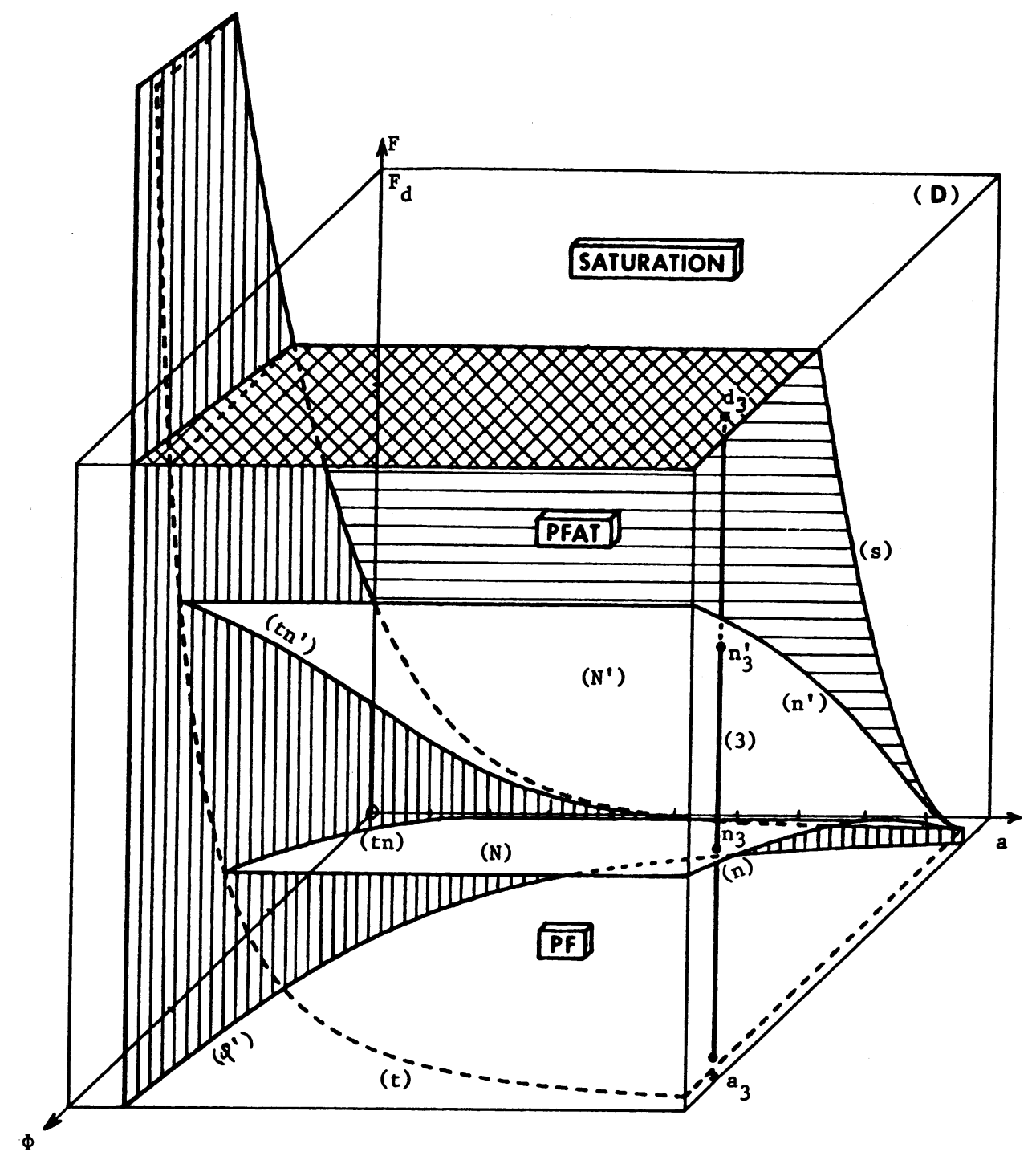

Fig. 9. - Synthetic representation, akin to that of figure 6, and showing the PF and PFAT ranges. Sheet (N) : $T=200 \mathrm{~K}$; sheet $\left(\mathrm{N}^{\prime}\right): T=500 \mathrm{~K}$.

beyond this limit for the following reasons. Firstly, such representation would not bring new essential information, apart the fact that there would exist a possibility of going directly from Poole regime to PFAT regime. Secondly, because this would need accounting for an eventual tunnelling through the inner barrier, a case also derived by Hill.

Now consider the line $a_{3} d_{3}$. It shows that when field is enhanced a Poole regime is followed by a PF regime which, beyond $\mathrm{n}_{3}(T=200 \mathrm{~K})$ or $\mathrm{n}_{3}^{\prime}(T=$ $500 \mathrm{~K}$ ) is overwhelmed by the PFAT regime. So it can be thought that, in this last region, a reinforced donor emptying happens, which in turn accelerates saturation settlement. Figure 9 shows that Hill's theory is liable to reduce very significantly, and especially in the low temperature range, the domain of effectiveness of a well characterized PF regime.

To come further into this matter, let us consider figure 10. In this figure, the respective variations of
$F_{a}(\Phi)$ and of $F_{\mathrm{t}}(a)$ were drawn in two connected diagrams. Curve $\left(n^{\prime \prime}\right)$ corresponding to $T=300 \mathrm{~K}$, was added to curves $(n)$ and $\left(n^{\prime}\right)$ of figure 9 . In addition, a second scaling in $\mathrm{N}_{\mathrm{d}}$ was represented, in order to bring into prominence the role played by the density of donors. It is then observed that, for a given density $N_{\mathrm{d}}$, curves (n), $\left(\mathrm{n}^{\prime}\right)$ and $\left(\mathrm{n}^{\prime \prime}\right)$ begin at the $F_{a}$ value corresponding to the horizontal line (h). This shows that Hill's theory would begin to apply for impurity depths the larger the lesser the temperature. Likewise, if $N_{\mathrm{d}}$ is too high, the PFAT effect cannot manifest itself in physically admissible ranges of $\Phi$ and $T$. Finally, it is observed that, for any given $\Phi\left(\Phi>\Phi_{\text {mini }}\right)$, the range of field strength over which PFAT is liable to work, is an increasing function of $\Phi$, at least when $F_{\mathrm{s}}<F_{\mathrm{d}}$ (segment $\mathrm{n}_{4} \mathrm{~s}_{4}$ for example).

Conversely, the right-hand side of figure 10 allows a straightforward delimitation of Poole, PF, and 


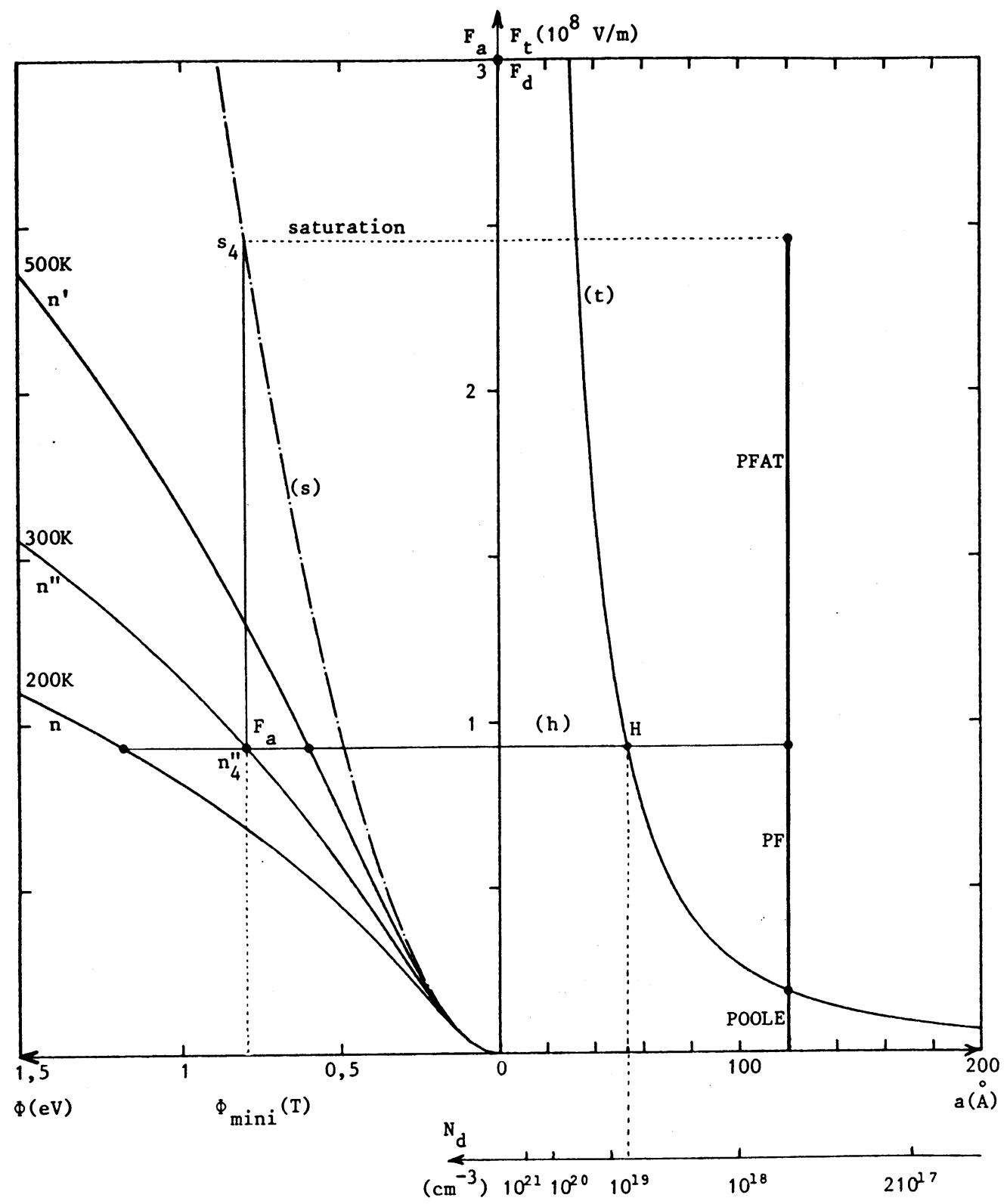

Fig. 10. - Variations of $F_{a}(\Phi)$ and $F_{\mathrm{t}}(a)$ in the same conditions and with notations like in figure 9. Intersection of sheet $\left(\mathrm{N}^{\prime \prime}\right)$, for $T=300 \mathrm{~K}$, with plane $\Phi F_{a}$ was added.

PFAT regimes. Effectively, any given $\Phi$ and $T$ define the horizontal line $(\mathrm{h})$ position, that is point $\mathrm{H}$. Now, for the particular density $N_{\mathrm{d}}$ corresponding to this point, Poole and PFAT regimes alone can exist. While for every lesser value of $N_{\mathrm{d}}$, the three regimes can exist as shown in the figure.

\section{Conclusion.}

Developments above allowed us to bring a synthetic survey of the Poole regime theory, and of the manner in which it is connected with PF regime theories. It became clear throughout, that some arbitrariness resides in the presently available approaches. This is the case of the model of isolated paired-wells, which principal merit is to afford the simplest way to succeed in mathematical treatments. But the model dodges somewhat the question of knowing how much nearer neighbours can be encompassed in mutual interactions. More basically, it can be asked how such interactions can be settled rationally, on a theoretical view-point.

Presently, our main purpose was to attempt to bring useful analyses, in view of experimental applications, by putting together the basic hypotheses as well as the involved physical modellings. In this prospect we tried to take out the weaknesses in definitions of the transition field from Poole to PF regimes, and to make more conspicuous the involved features. Likewise, we put forward the differences in 
authors' appreciation concerning, on the one hand the location in double wells of electrons to be liberated, and on the other hand the way by which they can be freed. We did also precise, throughout, some unpublished consequences of the existing theories. So, we showed, on quantitative bases, that Dussel and Boër approach leads to define a transition temperature $T_{\mathrm{t}}$, as a function of donor density (Fig. 3). We also examplified, through figure 8 , the large temperature dependence of the transition field $F_{a}$ between PF regime, and Hill's PFAT regime. Moreover, the manner in which the various fieldenhanced regimes are mutually connected with donor densities, well depths, and temperature, was displayed in numerical estimations in figure 10.
In such a prospect of experimental guidance, block-diagrams of figures 6 and 9 allowed us to bring some additional insight into the applicability of the available Poole and PF theories. In the same time, it brought us a means of visualizing fairly the influence of physical limitations, as well as space separations between the diverse field-enhanced bulk ionization processes. Moreover, it emphasizes sharply the importance of saturation, as well as of tunnelling.

Finally, as in addition some uncertainties concerning graphical representations was recalled as introductory remarks, the present work should bring to experimentalists circumstancial reasons of being aware of the inherent difficulties in putting together experimental data and PF-type models.

\section{References}

[1] Ongaro R., Pillonnet A., Revue Phys. Appl. 24 (1989) 1085.

[2] Hirai T., Nakada O., Jap. J. Appl. Phys. 7 (1968) 112-21.

[3] Pillonnet A., Thesis. Université Lyon 1, 1988.

[4] Connell G. A. N., Camphausen D. L., Paul W., Philos. Mag. 26 (1972) 541-51.

[5] Ieda M., Sawa G., Kato S., J. Appl. Phys. 42 (1971) 3737-40.

[6] Hartke J. L., J. Appl. Phys. 39 (1968) 4871-3.

[7] Conwell E. M., High field transport in semiconductors (Academic press. N.Y.) (1967).

[8] Hill R. M., Philos. Mag. 23 (1971) 59-86.

[9] Poole H. H., Philos. Mag. 32 (1916) 112-29.

[10] Feltz A., Kahnt H., Schirrmeister F., J. Phys. Colloque C4, Supplément $n^{\circ} 1042$ (1981) C4935-8.
[11] Boвe W., Fritzsch L., Phys. Status Solidi (b) 61 (1974) K35-9.

[12] Dussel G. A., Böer K. W., Phys. Status Solidi 39 (1970) 375-89.

[13] Vollmann W., Phys. Status Solidi (a) 22 (1974) 195203.

[14] Pillonnet A., Ongaro R., Revue Phys. Appl. 24 (1989) 109-16.

[15] Simmons J. G., Phys. Rev. 155 (1967) 657-60.

[16] Fritzsch L., Bobe W., Phys. Status Solidi (b) 58 (1973) K49-52.

[17] Arnett P. C., Klein N., J. Appl. Phys. 46 (1975) 1399-400.

[18] O'DWYER J. J., The theory of electrical conduction and breakdown in solid dielectrics (Clarendon Press) 1973. 